\title{
Use of Semantic Differential dimensions in concept learning'
}

Twenty-four Ss learned to sort words into two categories on the basis of conceptual dimensions (Evaluative and Potency) drawn from the Semantic Differential. Another group learned to categorize the same words under conditions wherein the concept was irrelevant and only rote memory could be used. The results indicate that $S$ s can easily identify and utilize the semantic dimensions (when available), and that these dimensions are suitable for use in concept-learning experiments.

Studies of concept learning almost universally employ abstract visual materials such as geometric designs as stimuli. These materials have the advantage that the stimulus characteristics are well known to, and discriminable by, S. However, they are also subject to the criticism of being somewhat artificial and unrepresentative of the verbal concepts typically used in everyday life.

Few studies have been conducted with meaningful verbal materials. Underwood and his associates (e.g., Underwood \& Richardson, 1956) have reported a series of concept studies using categories defined by the existence of low, medium, or high strength of association (dominance) between the category name and the verbal stimuli comprising the category. High dominance facilitated concept learning. Category membership in these studies was not based on common meanings, except insofar as common associations imply meaningful relationships.

Reed (1946) used a task in which $S$ had to classify sets of four nouns into categories named by nonsense syllables. There was no direct connotative relationship among the words in a set. Rather, word sets to which a particular nonsense syllable was the correct response all contained one word of a particular type (e.g., all GUZ sets contained a food word such as "turnip" or "apple"). Reed found that Ss set to look for a concept performed better than those merely instructed to memorize the word set-nonsense syllable pairings.

Aside from the work of Reed, there seems to have been little research in which word meanings are used to specify stimulus categories. There are three methodological difficulties in using connotative meaning in concept-learning studies. First, connotative meaning generally produces dichotomies rather than continuous dimensions. For example, most words are clearly either "food" words or "non-food" words. Second, meaningful words frequently carry some surpluṣ affective or associational meaning for Ss. Finally, the dimensionality of verbal materials is difficult to speci- fy, since a word may belong to an extremely large number of categories which may or may not be known to $\mathrm{S}$.

The Semantic Differential, introduced by Osgood and his co-workers (Osgood, Suci, \& Tannenbaum, 1957), provides an approach to verbal materials which may overcome these difficulties. Osgood et al have shown that there are three major dimensions of meaningfulness, Evaluative, Potency, and Activity. They are represented by the good-bad, hard-soft, and activepassive scales of the Semantic Differential, respectively, which are essentially factorially pure measures of the three major dimensions. These scales provide continuous dimensions of meaning of verbal material, and measure word positions on the dimensions with relatively high reliability. Further, the dimensions so scaled appear to be rather general in the population. Finally, they take into account the affective and associative aspects of words, as opposed to strict connotative meaning.

The purpose of this study was to establish the feasibility of using dimensions of meaning, as determined by the Semantic Differential, in concept-learning studies. To do so, it is necessary to demonstrate that the concepts represented by these dimensions can actually be identified and utilized by Ss. Specifically, it must be shown that Ss can learn to categorize words on the basis of these dimensions, and do so more efficiently than can be accounted for by simple rote learning.

Method

The experimental task required $S$ to learn the correct categorization of visually presented stimulus words. Eighty words, printed on $3 \times 5$ in. cards, were presented to $S$ one at a time with the request that he state whether the word was Type X or Type not-X. After each categorization, $S$ was told whether his choice was right or wrong. Upon completion of the list, the stimulus deck was shuffled, and $E$ went through the listagain in a new order. The list was presented three times, each time through the list counting as a block of 80 trials.

Two lists of words, representing the Evaluative (good-bad) and Potency (hard-soft) dimensions, were taken from the semantic atlas of Jenkins, Russell, \& Suci (1958). In each list, 40 words were drawn from each end of the scale (mean scale value between 1 and 3 , and between 5 and 7). One group of 24 Ss learned each list. The groups were subdivided into two learning conditions, Concept and No Concept. In the Concept condition, the $\mathrm{X}$ category consisted entirely of words rated between 6 and 7 , and the not-X category of words 
rated between 1 and 2. For the No Concept condition, the categories were scrambled so that each consisted of words from both ends of the dimension (e.g., both categories contained 20 good and 20 bad words). In the No Concept condition, then, the concept was irrelevant and $S$ could only rote-memorize the correct categorizations.

In a further subdivision, half of the Ss received instructions to memorize the correct categorization of each word, and the other half received instructions to discover the correct principle for categorization. The experiment was analyzed as a 2 by 2 by 2 by 3 repeated measures factorial design, with two relevant dimensions, two learning conditions, two kinds of instructions, and three successive blocks of 80 trials.

The Ss were 48 students from introductory psychology classes at Kansas State University. Each was recruited from an earlier concept-learning study, and was paid for participation.

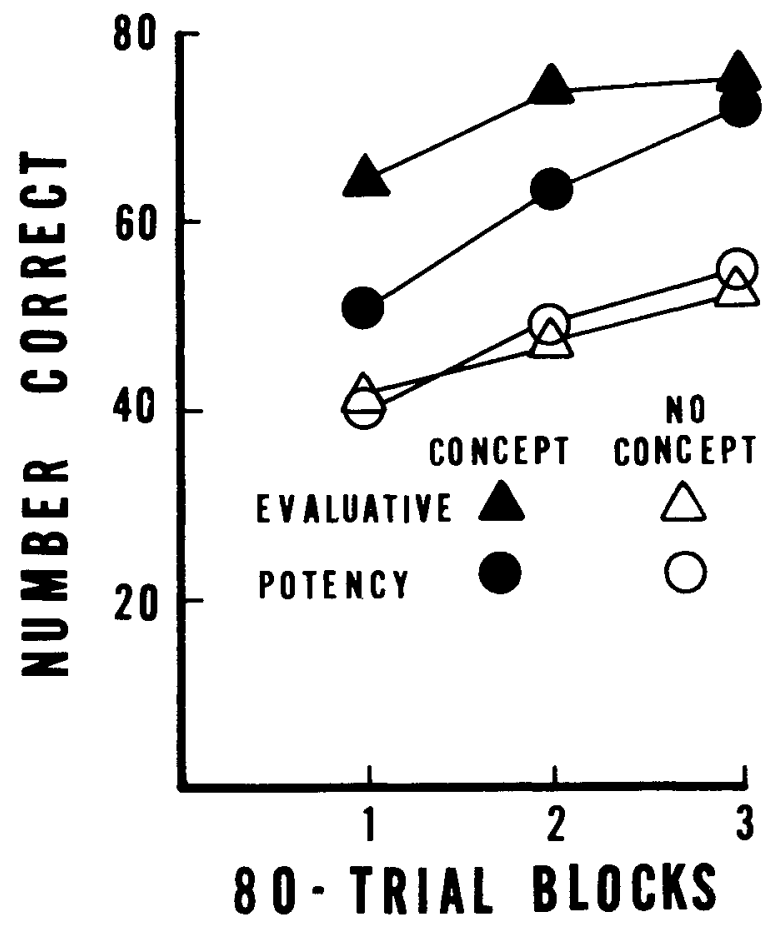

Fig. 1. Mean number of words categorized correctly in each 80-trial block for two word lists and two learning conditions. Each plotted point represents the mean of $12 \mathrm{Ss}$.

\section{Results}

The results are shown in Fig. 1. Performance of the Concept groups was significantly superior to that of the No Concept groups, $F=52.64, \mathrm{df}=1 / 40, \mathrm{p}<.01$. There was no significant difference in difficulty between the concepts, despite the appearance of the curves in Fig. 1. Instructions made no difference, probably because all Ss had participated in another concept-learning experiment. Learning occurred in both the Concept and No Concept conditions, and the overall improvement across blocks was highly significant, $F=70.18, \mathrm{df}=2 / 80$, $p<.01$. The slight difference in slope of the curves for the two dimensions proved to be marginally significant, $\mathrm{F}=4.09, \mathrm{df}=2 / 80, \mathrm{p}<.05$.

\section{Discussion}

It is clear from the results that the Concept groups are making use of the appropriate concepts, rather than simply rote memorizing the lists. The rapid grasp of the dimensions by most $\mathrm{Ss}$ in the Concept groups, even though no mention was made of the dimensions or the nature of the concepts involved, indicates that these dimensions are relatively obvious to most Ss. In some cases, Ss made only two or three errors, followed by over 75 consecutive correct responses on words seen for the first time. These results demonstrate that verbal dimensions drawn from the Semantic Differential can be used effectively in studies of concept learning; thus the way appears open to a variety of more meaningful studies of conceptual behavior. Strong support is also provided for Osgood's contention that Semantic Differential factors represent real dimensions of meaning to most individuals.

\section{References}

Jenkins, J. J., Russell, W. A. \& Suci, G. J. An atlas of semantic profiles for 360 words. Amer. J. Psychol., 1858, 71, 688-699.

Osgood, C. E., Suci, G. J., \& Tannenbaum, P. H. The measurement of meaning. Urbana: University of Illinois Press, 1957.

Reed, H. B. Factors influencing learning and retention of concepts. I. Influence of set. $J$. exp. Psychol., 1946, 36, 71-87.

Underwood, B. J., \& Richardson, J. Verbal concept learning as a function of instructions and dominance level. 1 . exp. Psychol., $1956,51,229-238$.

\section{Note}

1. This research was supported in part by a research grant (MH 11283) from the United States Public Health Service, National Institute of Mental Health. The writer is indebted to Pamela S. Carr and Curtis L. Taylor for assistance in collection and analysis of the data reported here. 